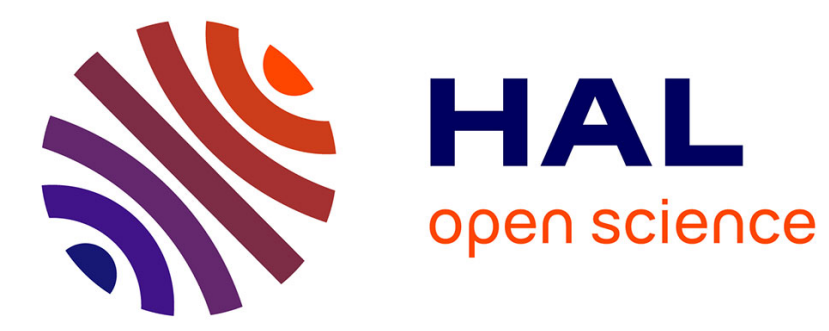

\title{
Dynamic Epistemic Logic as a Substructural Logic
}

Guillaume Aucher

\section{To cite this version:}

Guillaume Aucher. Dynamic Epistemic Logic as a Substructural Logic. Johan van Benthem on Logic and Information Dynamics, 5, pp.855 - 880, 2014, Outstanding Contributions to Logic, 10.1007/9783-319-06025-5_33. hal-01098793

\section{HAL Id: hal-01098793 \\ https://inria.hal.science/hal-01098793}

Submitted on 29 Dec 2014

HAL is a multi-disciplinary open access archive for the deposit and dissemination of scientific research documents, whether they are published or not. The documents may come from teaching and research institutions in France or abroad, or from public or private research centers.
L'archive ouverte pluridisciplinaire HAL, est destinée au dépôt et à la diffusion de documents scientifiques de niveau recherche, publiés ou non, émanant des établissements d'enseignement et de recherche français ou étrangers, des laboratoires publics ou privés. 


\title{
Dynamic Epistemic Logic as a Substructural Logic
}

\author{
Guillaume Aucher
}

\begin{abstract}
Dynamic Epistemic Logic (DEL) is an influential logical framework for reasoning about the dynamics of beliefs and knowledge. It has been related to older and more established logical frameworks. Despite these connections, DEL remains, arguably, a rather isolated logic in the vast realm of non-classical logics and modal logics. This is problematic if logic is to be viewed ultimately as a unified and unifying field and if we want to avoid that DEL goes on "riding off madly in all directions" (a metaphor used by van Benthem about logic in general). In this article, we show that DEL can be redefined naturally and meaningfully as a two-sorted substructural logic. In fact, it is even one of the most primitive substructural logics since it does not preserve any of the structural rules. Moreover, the ternary semantics of DEL and its dynamic interpretation provides a conceptual foundation for the Routley \& Meyer's semantics of substructural logics.
\end{abstract}

Key words: Dynamic Epistemic Logic, Substructural Logics, Update, Ternary Relation, Dynamic Inference

\section{Introduction}

Dynamic Epistemic Logic (DEL) is an influential logical framework for reasoning about the dynamics of beliefs and knowledge, which has drawn the attention of a number of researchers ever since the seminal publication of [17]. A number of contributions have linked DEL to older and more established logical frameworks: it has been embedded into (automata) PDL [40, 34], it has been given an algebraic semantics $[8,9]$, and it has been related to epistemic temporal logic $[32,6]$ and the situation calculus [31, 38]. Many of these links have been established by van Ben-

Guillaume Aucher

University of Rennes 1 - INRIA, 263, Avenue du Gnral Leclerc, 35042 Rennes Cedex, France e-mail: guillaume.aucher@irisa.fr 
them himself. Despite these connections, DEL remains, arguably, a rather isolated logic in the vast realm of non-classical logics and modal logics. This is problematic if logic is to be viewed ultimately as a unified and unifying field and if we want to avoid that DEL goes on "riding off madly in all directions" (a metaphor used by van Benthem $[28,30]$ about logic in general). In this article, we will show that DEL can be redefined naturally and meaningfully as a two-sorted substructural logic. In fact, it is even one of the most primitive substructural logics since it does not preserve any of the structural rules.

Substructural logics will also benefit from this interaction with DEL. The wellknown semantics for substructural logics is based on a ternary relation introduced by Routley \& Meyer for relevance logic in the 1970's [60, 61, 59, 62]. However, the introduction of this ternary relation was originally motivated by technical reasons, and it turns out that providing a non-circular and conceptually grounded interpretation of this relation remains problematic [18]. As we shall see, the ternary semantics of DEL provides a conceptual foundation for Routley \& Meyer's semantics. In fact, the dynamic interpretation induced by the DEL framework turns out to be not only meaningful, but also consistent with the interpretations of this ternary relation proposed in the substructural literature.

The article is structured as follows. In Section 2 we recall the core of DEL viewed from a semantic perspective. In Section 3 we briefly recall elementary notions of relevance and substructural logics and we observe that the ternary relation of relevance logic can be interpreted as a sort of update. In Section 4 we proceed further to define a substructural language based on this idea. This substructural language extends the DEL language with operators stemming from the Lambek calculus (a substructural logic), but we show that these different substructural operators actually correspond to the DEL operators of $[3,4]$. This allows us to show that DEL is a (two-sorted) substructural logic. In this section we also formally relate these operators to the dynamic inferences introduced by van Benthem [23]. In Section 5 we conclude and I give some personal views about the future of DEL and logical dynamics.

\section{Dynamic Epistemic Logic}

Dynamic epistemic logic (DEL) is a relatively recent non-classical logic [17] which extends ordinary modal epistemic logic [45] by the inclusion of event/action models (called $\mathscr{L}_{\alpha}$-models in this article) to describe actions, and a product update operator that defines how epistemic models (called $\mathscr{L}$-models in this article) are updated as the consequence of executing actions described through event models (see $[10,37,30]$ for more details). So, the methodology of DEL is such that it splits the task of representing the agents' beliefs and knowledge into three parts: first, one represents their beliefs/knowledge about an initial situation; second, one represents their beliefs/knowledge about an event taking place in this situation; third, one represents the way the agents update their beliefs/knowledge about the situation after 
(or during) the occurrence of the event. Following this methodology, we also split the exposition of the DEL framework into three sections.

\subsection{Representation of the Initial Situation: $\mathscr{L}$-model}

In the rest of this article, $A T M$ is a countable set of propositional letters called atomic facts which describe static situations, and $A G T:=\{1, \ldots, m\}$ is a finite set of agents.

Definition 1 (Language $\mathscr{L}$ and $\mathscr{L}$-structure). We define the language $\mathscr{L}$ inductively as follows:

$$
\mathscr{L}: \varphi::=p|\neg \varphi| \varphi \wedge \varphi|\varphi \vee \varphi| \square_{j} \varphi
$$

where $p$ ranges over $A T M$ and $j$ over $A G T$. We define $\perp:=p \wedge \neg p$ for a chosen $p \in A T M$ and we also define $T:=\neg \perp$. The formula $\diamond_{j} \varphi$ is an abbreviation for $\neg \square_{j} \neg \varphi$, the formula $\varphi \rightarrow \psi$ is an abbreviation for $\neg \varphi \vee \psi$, and the formula $\varphi \leftrightarrow \psi$ is an abbreviation for $(\varphi \rightarrow \psi) \wedge(\psi \rightarrow \varphi)$.

A $\mathscr{L}$-structure is defined inductively as follows, with $\varphi$ ranging over $\mathscr{L}$ :

$$
X::=\varphi \mid(X, X)
$$

We abusively write $\varphi \in X$ when the formula $\varphi \in \mathscr{L}$ is a substructure of $X$.

A (pointed) $\mathscr{L}$-model $(\mathscr{M}, w)$ represents how the actual world represented by $w$ is perceived by the agents. Atomic facts are used to state properties of this actual world.

Definition 2 ( $\mathscr{L}$-model). A $\mathscr{L}$-model is a tuple $\mathscr{M}=\left(W, R_{1}, \ldots, R_{m}, I\right)$ where:

- $W$ is a non-empty set of possible worlds,

- $R_{j} \subseteq W \times W$ is an accessibility relation on $W$, for each $j \in A G T$,

- $I: W \rightarrow 2^{A T M}$ is a function assigning to each possible world a subset of ATM. The function $I$ is called an interpretation.

We write $w \in \mathscr{M}$ for $w \in W$, and $(\mathscr{M}, w)$ is called a pointed $\mathscr{L}$-model $(w$ often represents the actual world). We denote by $\mathscr{C}$ the set of pointed $\mathscr{L}$-models. If $w, v \in$ $W$, we write $w R_{j} v$ or $(\mathscr{M}, w) R_{j}(\mathscr{M}, v)$ for $(w, v) \in R_{j}$, and $R_{j}(w)$ denotes the set $\left\{v \in W \mid w R_{j} v\right\}$.

Intuitively, $w R_{j} v$ means that in world $w$ agent $j$ considers that world $v$ might correspond to the actual world. Then, we define the following epistemic language that can be used to describe and state properties of $\mathscr{L}$-models:

Definition 3 (Truth conditions of $\mathscr{L}$ ). Let $\mathscr{M}$ be a $\mathscr{L}$-model, $w \in \mathscr{M}$ and $\varphi \in \mathscr{L}$. $\mathscr{M}, w \models \varphi$ is defined inductively as follows: 


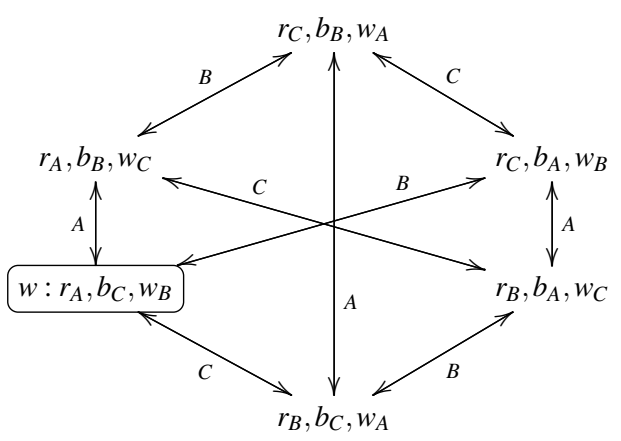

Fig. 1 Cards Example

$$
\begin{array}{llll}
\mathscr{M}, w \models p & \text { iff } & & p \in I(w) \\
\mathscr{M}, w \models \neg \psi & \text { iff } & \text { not } \mathscr{M}, w \models \varphi \\
\mathscr{M}, w \models \varphi \wedge \psi & \text { iff } & \mathscr{M}, w \models \varphi \text { and } \mathscr{M}, w \models \psi \\
\mathscr{M}, w \models \varphi \vee \psi & \text { iff } & \mathscr{M}, w \models \varphi \text { or } \mathscr{M}, w \models \psi \\
\mathscr{M}, w \models \square_{j} \varphi & \text { iff } & \text { for all } v \in R_{j}(w), \mathscr{M}, v \models \varphi
\end{array}
$$

We write $\mathscr{M} \models \varphi$ when $\mathscr{M}, w \models \varphi$ for all $w \in \mathscr{M}$, and $\models \varphi$ when for all $\mathscr{L}$-model $\mathscr{M}, \mathscr{M} \models \varphi$. A $\mathscr{L}$-formula $\varphi$ is said to be valid if $\models \varphi$. We extend the scope of the relation $\models$ to also relate pointed $\mathscr{L}$-models to structures:

$$
\mathscr{M}, w \models X, Y \quad \text { iff } \quad \mathscr{M}, w \models X \text { and } \mathscr{M}, w \models Y
$$

Let $C$ be a class of pointed $\mathscr{L}$-models, let $X, Y$ be $\mathscr{L}$-structures. We say that $X$ entails $Y$ in the class $C$, written $\left.X\right|_{\bar{C}} Y$, when the following holds:

$X \models_{\bar{C}} Y \quad$ iff $\quad$ for all pointed $\mathscr{L}$-model $(\mathscr{M}, w) \in C$, if for all $\varphi \in X \mathscr{M}, w \models \varphi$, then there is $\psi \in Y$ such that $\mathscr{M}, w \models \psi$.

We also write $X \models Y$ for $\left.X\right|_{\mathscr{C}} Y$, where $\mathscr{C}$ is the class of all pointed $\mathscr{L}$-models.

The formula $\square_{j} \varphi$ reads as "agent $j$ believes $\varphi$ ". Its truth conditions are defined in such a way that agent $j$ believes $\varphi$ is true in a possible world when $\varphi$ holds in all the worlds agent $j$ considers possible.

Example 1. Assume that agents A, B and C play a card game with three cards: a white one, a red one and a blue one. Each of them has a single card but they do not know the cards of the other players. At each step of the game, some of the players show their/her/his card to another player or to both other players, either privately or publicly. We want to study and represent the dynamics of the agents' beliefs/knowledge in this game. The initial situation is represented by the pointed $\mathscr{L}$-model $(\mathscr{M}, w)$ of Figure 1. 
In this example, $A G T:=\{A, B, C\}$ and $A T M:=\left\{r_{j}, b_{j}, w_{j} \mid j \in A G T\right\}$ where $r_{j}$ stands for 'agent $j$ has the $r$ ed card', $b_{j}$ stands for 'agent $\mathrm{j}$ has the $b$ lue card' and $w_{j}$ stands for 'agent $j$ has the $w$ hite card'. The boxed possible world corresponds to the actual world. The propositional letters not mentioned in the possible worlds do not hold in these possible worlds. The accessibility relations are represented by arrows indexed by agents between possible worlds. Reflexive arrows are omitted in the figure, which means that for all worlds $v \in \mathscr{M}$ and all agents $j \in A G T, v \in R_{j}(v)$. In this model, we have for example the following statement: $\mathscr{M}, w \models\left(w_{B} \wedge \neg \square_{A} w_{B}\right) \wedge$ $\square_{C} \neg \square_{A} w_{B}$. It states that player A does not 'know' that player B has the white card and player $\mathrm{C}$ 'knows' it.

\subsection{Representation of the Event: $\mathscr{L}_{\alpha}$-model}

The language $\mathscr{L}_{\alpha}$ was introduced in [11]. The propositional letters $p_{\psi}$ describing events are called atomic events and range over $A T M_{\alpha}=\left\{p_{\psi} \mid \psi\right.$ ranges over $\left.\mathscr{L}\right\}$. The reading of $p_{\psi}$ is "an event of precondition $\psi$ is occurring".

Definition 4 (Language $\mathscr{L}_{\alpha}$ and $\mathscr{L}_{\alpha}$-structure). We define the language $\mathscr{L}_{\alpha}$ inductively as follows:

$$
\mathscr{L}_{\alpha}: \alpha::=p_{\psi}|\neg \alpha| \alpha \wedge \alpha|\alpha \vee \alpha| \square_{j} \alpha
$$

where $\psi$ ranges over $\mathscr{L}$ and $j$ over $A G T$. We define $\perp:=p_{\psi} \wedge \neg p_{\psi}$ for a chosen $\psi \in \mathscr{L}$ and we define $\top:=\neg \perp$. The formula $\nabla_{j} \alpha$ is an abbreviation for $\neg \square_{j} \neg \alpha$, the formula $\alpha \rightarrow \beta$ is an abbreviation for $\neg \alpha \vee \beta$, and the formula $\alpha \leftrightarrow \beta$ is an abbreviation for $(\alpha \rightarrow \beta) \wedge(\beta \rightarrow \alpha)$.

A $\mathscr{L}_{\alpha}$-structure is defined inductively as follows, with $\beta$ ranging over $\mathscr{L}_{\alpha}$ :

$$
\mathscr{S}_{\alpha}: X_{\alpha}::=\beta \mid\left(X_{\alpha}, X_{\alpha}\right)
$$

We abusively write $\alpha \in X_{\alpha}$ when the formula $\alpha \in \mathscr{L}_{\alpha}$ is a substructure of $X_{\alpha}$.

A pointed $\mathscr{L}_{\alpha}$-model $(\mathscr{E}, e)$ represents how the actual event represented by $e$ is perceived by the agents. Intuitively, $f \in R_{j}(e)$ means that while the possible event represented by $e$ is occurring, agent $j$ considers possible that the possible event represented by $f$ is actually occurring.

Definition 5 ( $\mathscr{L}_{\alpha}$-model, [17]). A $\mathscr{L}_{\alpha}$-model is a tuple $\mathscr{E}=\left(W_{\alpha}, R_{1}, \ldots, R_{m}, I\right)$ where:

- $W_{\alpha}$ is a non-empty set of possible events,

- $R_{j} \subseteq W_{\alpha} \times W_{\alpha}$ is an accessibility relation on $W_{\alpha}$, for each $j \in A G T$,

- $I: W_{\alpha} \rightarrow \mathscr{L}$ is a function assigning to each possible event a formula of $\mathscr{L}$. The function $I$ is called the precondition function.

Let $P$ be a subset of $\mathscr{L}$. A $P$-complete $\mathscr{L}_{\alpha}$-model is a $\mathscr{L}_{\alpha}$-model which satisfies moreover the following condition: 


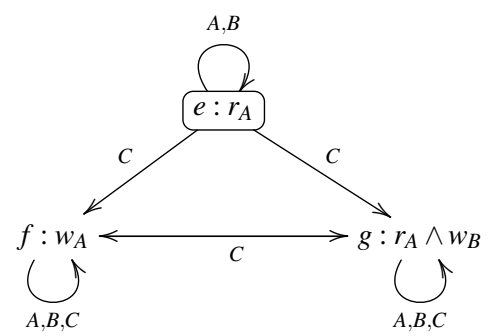

Fig. 2 Players A and B show their cards to each other in front of player C

- $I(e) \in P$, for each $e \in W_{\alpha}$

(P-complete)

We write $e \in \mathscr{E}$ for $e \in W_{\alpha}$, and $(\mathscr{E}, e)$ is called a pointed $\mathscr{L}_{\alpha}$-model ( $e$ often represents the actual event). We denote by $\mathscr{C}_{\alpha}$ the set of pointed $\mathscr{L}_{\alpha}$-models, by $\mathscr{C}_{\alpha}^{P}$ the set of pointed $P$-complete event models. If $e, f \in W_{\alpha}$, we write $e R_{j} f$ or $(\mathscr{E}, e) R_{j}(\mathscr{E}, f)$ for $(e, f) \in R_{j}$, and $R_{j}(e)$ denotes the set $\left\{f \in W_{\alpha} \mid e R_{j} f\right\}$.

The truth conditions of the language $\mathscr{L}_{\alpha}$ are identical to the truth conditions of the language $\mathscr{L}$ :

Definition 6 (Truth conditions of $\mathscr{L}_{\alpha}$ ). Let $\mathscr{E}$ be a $\mathscr{L}_{\alpha}$-model, $e \in \mathscr{E}$ and $\alpha \in \mathscr{L}_{\alpha}$. $\mathscr{E}, e \models \alpha$ is defined inductively as follows:

$$
\begin{array}{lll}
\mathscr{E}, e=p_{\psi} & \text { iff } & I(e)=\psi \\
\mathscr{E}, e=\neg \alpha & \text { iff } & \text { not } \mathscr{E}, e=\alpha \\
\mathscr{E}, e=\alpha \wedge \beta & \text { iff } & \mathscr{E}, e=\alpha \text { and } \mathscr{E}, e=\beta \\
\mathscr{E}, e=\alpha \vee \beta & \text { iff } & \mathscr{E}, e=\alpha \text { or } \mathscr{E}, e=\beta \\
\mathscr{E}, e=\square_{j} \alpha & \text { iff } & \text { for all } f \in R_{j}(e), \mathscr{E}, f \models \alpha
\end{array}
$$

Let $C$ be a class of pointed $\mathscr{L}_{\alpha}$-models, let $X_{\alpha}, Y_{\alpha}$ be $\mathscr{L}_{\alpha}$-structures. We say that $X$ entails $Y$ in the class $C$, written $\left.X_{\alpha}\right|_{\bar{C}} Y_{\alpha}$, when the following holds:

$X_{\alpha} \models_{\bar{C}} Y_{\alpha} \quad$ iff $\quad$ for all pointed $\mathscr{L}_{\alpha}$-model $(\mathscr{E}, e) \in C$,

if for all $\alpha \in X_{\alpha} \mathscr{E}, e \models \alpha$, then there is $\beta \in Y_{\alpha}$ such that $\mathscr{E}, e \models \beta$.

We also write $X_{\alpha}=Y_{\alpha}$ for $X_{\alpha} \frac{\overline{\mathscr{C}}_{\alpha}}{{ }} Y_{\alpha}$, where $\mathscr{C}_{\alpha}$ is the class of all pointed $\mathscr{L}_{\alpha-}$ models.

Example 2. Let us resume Example 1 and assume that players A and B show their card to each other. As it turns out, C noticed that A showed her card to B but did not notice that B did so to A. Players A and B know this. This event is represented in the $\mathscr{L}_{\alpha}$-model $(\mathscr{E}, e)$ of Figure 2 . The boxed possible event $e$ corresponds to the actual event 'player A shows her red card' (with precondition $r_{A}$ ), $f$ stands for the event 'player A shows her white card' (with precondition $w_{A}$ ) and $g$ stands for the atomic event "players A and B show their red and white cards respectively to each 


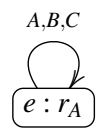

Fig. 3 Public announcement $\mathscr{L}_{\alpha}$-model of $r_{A}$

other' (with precondition $r_{A} \wedge w_{B}$ ). The following statement holds in the example of Figure 2:

$$
\begin{aligned}
\mathscr{E}, e \models p_{r_{A}} & \wedge\left(\nabla_{A} p_{r_{A}} \wedge \square_{A} p_{r_{A}}\right) \wedge\left(\diamond_{B} p_{r_{A}} \wedge \square_{B} p_{r_{A}}\right) \\
& \wedge\left(\nabla_{C} p_{w_{A}} \wedge \nabla_{C} p_{r_{A} \wedge w_{A}} \wedge \square_{C}\left(p_{w_{A}} \vee p_{r_{A} \wedge w_{A}}\right)\right)
\end{aligned}
$$

It states that players A and B show their cards to each other, players A and B 'know' this and consider it possible, while player $\mathrm{C}$ considers possible that player A shows her white card and also considers possible that player A shows her red card, since he does not know her card. In fact, that is all that player $\mathrm{C}$ considers possible since he believes that either player A shows her red card or her $w$ hite card. Another example of $\mathscr{L}_{\alpha}$-model is given in Figure 3 . This second example corresponds to the event whereby Players A shows her card publicly to everybody.

The following statement holds in the example of Figure 3:

$$
\begin{aligned}
\mathscr{E}, e=p_{r_{A}} & \wedge \square_{A} p_{r_{A}} \wedge \square_{B} p_{r_{A}} \wedge \square_{C} p_{r_{A}} \\
& \wedge \square_{A} \square_{A} p_{r_{A}} \wedge \square_{A} \square_{B} p_{r_{A}} \wedge \square_{A} \square_{C} p_{r_{A}} \\
& \wedge \square_{B} \square_{A} p_{r_{A}} \wedge \square_{B} \square_{B} p_{r_{A}} \wedge \square_{B} \square_{C} p_{r_{A}} \\
& \wedge \square_{C} \square_{A} p_{r_{A}} \wedge \square_{C} \square_{B} p_{r_{A}} \wedge \square_{C} \square_{C} p_{r_{A}} \\
& \wedge \ldots
\end{aligned}
$$

It states that player A shows her red card and that players A, B and C 'know' it, that players A, B and C 'know' that each of them 'know' it, etc... in other words, there is common knowledge among players $\mathrm{A}, \mathrm{B}$ and $\mathrm{C}$ that player A shows her red card. $^{1}$

$$
\mathscr{E}, e \models p_{r_{A}} \wedge \square_{A G T}^{*} p_{r_{A}}
$$

\subsection{Update of the Initial Situation by the Event: Product Update}

The DEL product update of [17] is defined as follows. This update yields a new $\mathscr{L}$ model $(\mathscr{M}, w) \otimes(\mathscr{E}, e)$ representing how the new situation which was previously

${ }^{1}$ We write $\mathscr{E}, e \models \square_{A G T}^{*} \alpha$ when for all $f \in\left(\bigcup_{j \in A G T} R_{j}\right)^{*}(e), \mathscr{E}, f \models \alpha$. See for example [41] for a detailed study of the operator $\square_{A G T}^{*}$ of common knowledge 


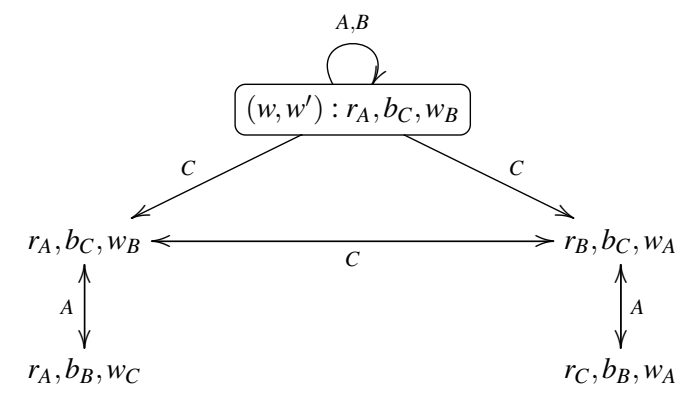

Fig. 4 Situation after the update of the situation represented in Figure 1 by the event represented in Figure 2

represented by $(\mathscr{M}, w)$ is perceived by the agents after the occurrence of the event represented by $(\mathscr{E}, e)$.

Definition 7 (Product update). Let $(\mathscr{M}, w)=\left(W, R_{1}, \ldots, R_{m}, I, w\right)$ be a pointed $\mathscr{L}$-model and let $(\mathscr{E}, e)=\left(W_{\alpha}, R_{1}, \ldots, R_{m}, I, e\right)$ be a pointed $\mathscr{L}_{\alpha}$-model such that $\mathscr{M}, w \models I(e)$. The product update of $(\mathscr{M}, w)$ and $(\mathscr{E}, e)$ is the pointed $\mathscr{L}$-model $(\mathscr{M} \otimes \mathscr{E},(w, e))=\left(W^{\otimes}, R_{1}^{\otimes}, \ldots, R_{m}^{\otimes}, I^{\otimes},(w, e)\right)$ defined as follows: for all $v \in W$ and all $f \in W_{\alpha}$,

- $W^{\otimes}=\left\{(v, f) \in W \times W_{\alpha} \mid \mathscr{M}, v \models I(f)\right\}$,

- $R_{j}^{\otimes}(v, f)=\left\{(u, g) \in W^{\otimes} \mid u \in R_{j}(v)\right.$ and $\left.g \in R_{j}(f)\right\}$,

- $I^{\otimes}(v, f)=I(v)$.

Example 3. As a result of the event described in Example 2, the agents update their beliefs. We get the situation represented in the $\mathscr{L}$-model $(\mathscr{M}, w) \otimes(\mathscr{E}, e)$ of Figure 4. In this $\mathscr{L}$-model, we have for example the following statement:

$$
(\mathscr{M}, w) \otimes(\mathscr{E}, e) \models\left(w_{B} \wedge \square_{A} w_{B}\right) \wedge \square_{C} \neg \square_{A} w_{B} .
$$

It states that player A 'knows' that player B has the white card but player $\mathrm{C}$ believes that it is not the case.

\section{Substructural Logics}

Substructural logics are a family of logics lacking some of the structural rules of classical logic. A structural rule is a rule of inference which is closed under substitution of formulas. The structural rules for classical logic are given in Figure 5 (U,X,Y,Z denote $\mathscr{L}$-structures). While (Weakening) and (Contraction) are often dropped like in relevance logic and linear logic, the rule of (Associativity) is often preserved. We shall see in this article that DEL invalidates all of them. 


$$
\begin{array}{lll}
\text { Weakening: } & \text { Associativity: } \\
\frac{Y \models Z}{X, Y \models Z} W_{L} & \frac{Y \models Z}{Y \models Z, X} W_{R} & \frac{X,(Y, Z) \models U}{(X, Y), Z \models U} B \\
\text { Contraction: } & \text { Commutativity: } \\
\frac{(X, X), Y \models Z}{X, Y \models Z} C_{L} \frac{Y \models Z,(X, X)}{Y \models Z, X} C_{R} & \frac{Y, X \models Z}{X, Y \models Z} C
\end{array}
$$

Fig. 5 Structural rules of classical logic

\subsection{A Substructural Language}

Our exposition of substructural logics is based on [57, 58, 39]. The logical framework presented in [57] is much more general and studies a wide range of substructural logics: relevance logic, linear logic, lambek calculus, display logic, etc. .. For what concerns us in this article, we will only introduce a fragment of this general framework. The semantics of this fragment is based on the ternary relation of the frame semantics for relevant logic originally introduced by Routley \& Meyer $[60,61,59,62]$. Another semantics proposed independently by Urquhart $[65,63,64]$ at about the same time will be discussed at the end of this section.

Definition 8 (Language $\mathscr{L}_{\text {Sub }}$ and $\mathscr{L}_{\text {Sub-structure). The language }} \mathscr{L}_{\text {Sub }}$ is defined inductively as follows:

$$
\begin{aligned}
& \mathscr{L}_{\text {Sub }}: \varphi::=\top|\perp| p|\neg \varphi| \varphi \wedge \varphi|\varphi \vee \varphi| \square \varphi \mid \\
& \varphi \supset \varphi|\varphi \subset \varphi| \varphi \circ \varphi
\end{aligned}
$$

where $p$ ranges over $A T M$.

A $\mathscr{L}_{\text {Sub }}$-structure is defined inductively as follows, with $\varphi$ ranging over $\mathscr{L}_{\text {Sub }}$ :

$$
X::=\varphi|(X, X)|(X ; X)
$$

Definition 9 (Point set, plump accessibility relation). A point set $\mathscr{P}=(P, \sqsubseteq)$ is a set $P$ together with a partial order $\sqsubseteq$ on $P$. The set $\operatorname{Prop}(\mathscr{P})$ of propositions on $\mathscr{P}$ is the set of all subsets $X$ of $P$ which are closed upwards: that is, if $x \in X$ and $x \sqsubseteq x^{\prime}$ then $x^{\prime} \in X$. We abusively write $x \in \mathscr{P}$ for $x \in P$.

- A binary relation $\mathscr{S}$ is a positive two-place accessibility relation on the point set $\mathscr{P}$ iff for any $x, y \in \mathscr{P}$ where $x \mathscr{S} y$, if $x^{\prime} \sqsubseteq x$ then there is a $y^{\prime} \sqsupseteq y$, where $x^{\prime} \mathscr{S} y^{\prime}$. Similarly, if $x \mathscr{S} y$ and $y \sqsubseteq y^{\prime}$ then there is some $x^{\prime} \sqsubseteq x$, where $x^{\prime} \mathscr{S} y^{\prime}$.

- A ternary relation $\mathscr{R}$ is a three-place accessibility relation iff whenever $\mathscr{R} x y z$ and $z \sqsubseteq z^{\prime}$ then there are $y^{\prime} \sqsupseteq y$ and $x^{\prime} \sqsubseteq x$, where $\mathscr{R} x^{\prime} y^{\prime} z^{\prime}$. Similarly, if $x^{\prime} \sqsubseteq x$ then 
there are $y^{\prime} \sqsubseteq y$ and $z^{\prime} \sqsupseteq z$, where $\mathscr{R} x^{\prime} y^{\prime} z^{\prime}$, and if $y^{\prime} \sqsubseteq y$ then there are $x^{\prime} \sqsubseteq x$ and $z^{\prime} \sqsupseteq z$, where $\mathscr{R} x^{\prime} y^{\prime} z^{\prime}$.

- A ternary relation $\mathscr{R}$ is a plump accessibility relation on the point set $\mathscr{P}$ if and only if for any $x, y, z, x^{\prime}, y^{\prime}, z^{\prime} \in \mathscr{P}$ such that $\mathscr{R} x y z$, if $x^{\prime} \sqsubseteq x, y^{\prime} \sqsubseteq y$ and $z \sqsubseteq z^{\prime}$, then $\mathscr{R} x^{\prime} y^{\prime} z^{\prime}$.

Our definition of $\mathscr{L}_{\text {Sub }}$-model corresponds to the definition of a model in [57, Chap. 11] stripped out from all its truth sets. These other features are not needed for what concerns us here.

Definition 10 ( $\mathscr{L}_{\text {Sub }}-$ model). A $\mathscr{L}_{\text {Sub }}$-model is a tuple $\mathscr{M}_{\mathscr{R}}=(\mathscr{P}, \mathscr{S}, \mathscr{R}, \mathscr{I})$ where:

- $\mathscr{P}=(P, \sqsubseteq)$ is a point set;

- $\mathscr{S} \subseteq \mathscr{P} \times \mathscr{P}$ is a positive two-place accessibility relation on $\mathscr{P}$;

- $\mathscr{R} \subseteq \mathscr{P} \times \mathscr{P} \times \mathscr{P}$ is a three-place accessibility relation on $\mathscr{P}$;

- $\mathscr{I}: P \rightarrow 2^{A T M}$ is an interpretation function.

We abusively write $x \in \mathscr{M}_{\mathscr{R}}$ for $x \in \mathscr{P}$, and $\left(\mathscr{M}_{\mathscr{R}}, x\right)$ is called a pointed $\mathscr{L}_{\text {Sub }}-$ model.

Note that in the above definition, there could be multiple positive two-place accessibility relations $\mathscr{S}_{1}, \ldots, \mathscr{S}_{n}$ corresponding to multiple modalities $\square_{1}, \ldots \square_{n}$. We refrain from defining $\mathscr{L}_{\text {Sub }}$-models in their full generality in order to ease the readability of the article.

Definition 11 (Truth conditions of $\mathscr{L}_{\text {Sub }}$ ). Let $\mathscr{M}_{\mathscr{R}}$ be a $\mathscr{L}_{\text {Sub }}$-model, $x \in \mathscr{M}_{\mathscr{R}}$ and $\varphi \in \mathscr{L}_{\text {Sub }}$. The relation $\mathscr{M}_{\mathscr{R}}, x \Vdash \varphi$ is defined inductively as follows:

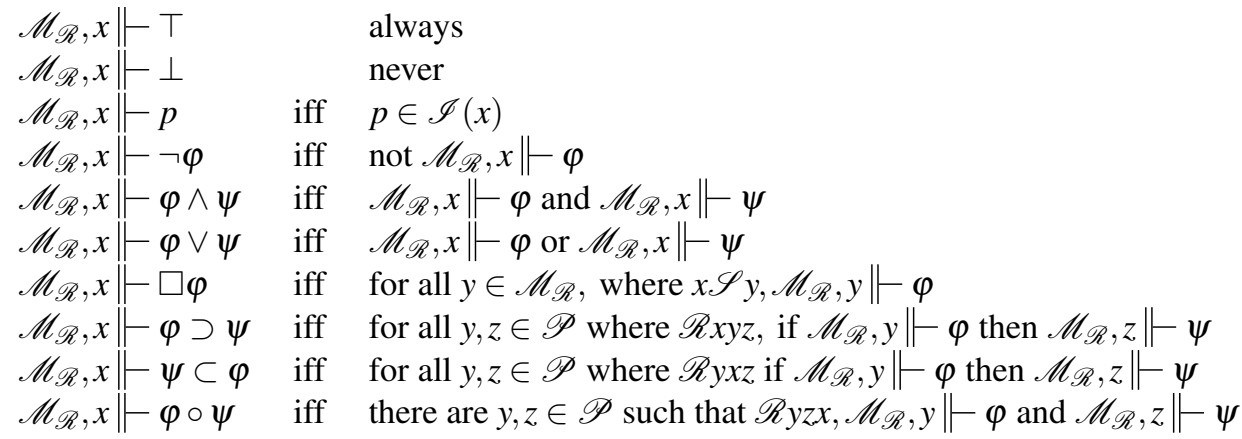

We extend the scope of the relation $\|$ to also relate points to $\mathscr{L}_{\text {Sub }}$-structures:

$\mathscr{M}_{\mathscr{R}}, x \| X, Y \quad$ iff $\quad \mathscr{M}_{\mathscr{R}}, x \| X$ and $\mathscr{M}_{\mathscr{R}}, x \| Y$

$\mathscr{M}_{\mathscr{R}}, x \|-X ; Y \quad$ iff $\quad$ there are $y, z \in \mathscr{M}_{\mathscr{R}}$ such that $\mathscr{R} y z x, \mathscr{M}_{\mathscr{R}}, y \|-X$ and $\mathscr{M}_{\mathscr{R}}, z \| Y$

We say that $\mathscr{M}_{\mathscr{R}}$ validates a $\mathscr{L}_{\text {Sub }}$-structure $X$ when for all $x \in \mathscr{M}_{\mathscr{R}}, \mathscr{M}_{\mathscr{R}}, x \| X$. Let $X$ be a structure and let $\varphi \in \mathscr{L}_{\text {Sub }}$. We say that $X$ entails $\varphi$, written $X \| \varphi$, when the following holds: 
$X \| \varphi \quad$ iff $\quad$ for all pointed $\mathscr{L}_{\text {Sub }}-\operatorname{model}\left(\mathscr{M}_{\mathscr{R}}, x\right)$, if $\mathscr{M}_{\mathscr{R}}, x \Vdash X$, then $\mathscr{M}_{\mathscr{R}}, x \| \varphi$.

Note that unlike many substructural logics, we use a Boolean negation. We list below some key inferences of substructural logics, more precisely of the Lambek Calculus:

$$
\begin{array}{rll}
\varphi ; \psi \| \chi & \text { iff } & \varphi \Vdash \psi \supset \chi \\
\varphi \Vdash \psi \supset \chi & \text { iff } & \varphi \circ \psi \| \chi \\
\varphi \circ \psi \| \chi & \text { iff } & \psi \| \chi \subset \varphi \\
\varphi \Vdash \psi \supset \chi & \text { iff } & \psi \| \chi \subset \varphi
\end{array}
$$

\section{Urquhart's semantics}

The Urquhart's semantics for relevance logic was developed independently from the Routley \& Meyer's semantics in the early 1970's. An operational frame is a set of points $\mathscr{P}$ together with a function which gives us a new point from a pair of points:

$$
\sqcup: \mathscr{P} \times \mathscr{P} \rightarrow \mathscr{P} .
$$

An operational model is then an operational frame together with a relation $\|$ which indicates what formulas are true at what points. The truth conditions for the implication $\supset$ are defined as follows:

$$
x \Vdash \varphi \supset \psi \text { iff for each } y \text {, if } y \Vdash \varphi \text { then } x \sqcup y \| \psi
$$

As one can easily notice, an operational frame is a Routley \& Meyer frame where $\mathscr{R} x y z$ holds if and only if $x \sqcup y=z$. Hence, the ternary relation $\mathscr{R}$ of the Routley \& Meyer semantics is a generalization of the function $\sqcup$ of the Urquhart's semantics. Because it is a relation, it allows moreover to apply $x$ to $y$ and yield either a set of outcomes or no outcome at all.

\subsection{Updates as Ternary Relations}

The ternary relation $\mathscr{R}$ of the Routley \& Meyer semantics was introduced originally for technical reasons: any 2-ary ( $n$-ary) connective of a logical language can be given a semantics by resorting to a 3 -ary (resp. $n+1$-ary) relation on worlds. Subsequently, a number of philosophical interpretations of this ternary relation have been proposed and we will briefly recall some of them at the end of this section (see [18, 58, 50] for more details). However, one has to admit that providing a non-circular and conceptually grounded interpretation of this relation remains problematic. In this article, we propose a new dynamic interpretation of this relation, inspired by the ternary semantics of DEL. 
First, one should observe that the DEL product update $\otimes$ of Definition 7 can be seen as a partial function $\mathscr{F}$ from a pair of pointed $\mathscr{L}$-model and pointed $\mathscr{L}_{\alpha-}$ model to another pointed $\mathscr{L}$-model:

$$
\mathscr{F}: \mathscr{C} \times \mathscr{C}_{\alpha} \rightarrow \mathscr{C}
$$

There is a formal similarity between this abstract definition of the DEL product update and the function $\sqcup$ of Equation (6) introduced by Urquhart in the early 1970s for providing a semantics to the implication of relevance logic. This similarity is not only formal but also intuitively meaningful. Indeed, the intuitive interpretation of the DEL product update operator is very similar to the intuitive interpretation of the function $\sqcup$ of Urquhart. Points are sometimes also called "worlds", "states", "situation", "set-ups", and as explained by Restall:

"We have a class of points (over which $x$ and $y$ vary), and a function $\sqcup$ which gives us new points from old. The point $x \sqcup y$ is supposed, on Urquhart's interpretation, to be the body of information given by combining $x$ with $y$."

[58, p. 363]

and also, keeping in mind the truth conditions for the connective $\supset$ of Equation (7):

"To be committed to $A \supset B$ is to be committed to $B$ whenever we gain the information that $A$. To put it another way, a body of information warrants $A \supset B$ if and only if whenever you update that information with new information which warrants $A$, the resulting (perhaps new) body of information warrants $B$. ." (my emphasis)

$[58$, p. 362]

From these two quotes, it is natural to interpret the DEL product update $\otimes$ of Definition 7 as a specific kind of Urquhart's function $\sqcup$ (Equation (6)). Moreover, as explained by Restall, this substructural "update" can be nonmonotonic and may correspond to some sort of revision:

"[C]ombination is sometimes nonmonotonic in a natural sense. Sometimes when a body of information is combined with another body of information, some of the original body of information might be lost. This is simplest to see in the case motivating the failure of $A \vdash B \supset A$. A body of information might tell us that $A$. However, when we combine it with something which tells us $B$, the resulting body of information might no longer warrant $A$ (as $A$ might with $B$ ). Combination might not simply result in the addition of information. It may well warrant its revision." (my emphasis)

[58, p. 363]

Our dynamic interpretation of the ternary relation is consistent with the above considerations: sometimes, updating beliefs amounts to revise beliefs. As it turns out, belief revision has also been extensively studied within the DEL framework and DEL has been extended to deal with this phenomenon [1, 36, 26, 13, 14, 46, 2].

More generally, an update can be seen as a partial function $\mathscr{F}$ from a pair of pointed $\mathscr{L}$-model and pointed $\mathscr{L}_{\alpha}$-model to a set of pointed $\mathscr{L}$-model:

$$
\mathscr{F}: \mathscr{C} \times \mathscr{C}_{\alpha} \rightarrow \mathscr{P}(\mathscr{C})
$$

Equivalently, an update can be seen as a ternary relation $\mathscr{R}$ defined on $\mathscr{C} \cup \mathscr{C} \alpha$ between three pointed models $\left((\mathscr{M}, w),(\mathscr{E}, e),\left(\mathscr{M}_{f}, w_{f}\right)\right)$ where $(\mathscr{M}, w)$ is a pointed $\mathscr{L}$-model, $(\mathscr{E}, e)$ is a pointed $\mathscr{L}_{\alpha}$-model and $\left(\mathscr{M}_{f}, w_{f}\right)$ is another pointed $\mathscr{L}$ model: 


$$
\mathscr{R} \subseteq \mathscr{C} \times \mathscr{C}_{\alpha} \times \mathscr{C}
$$

The ternary relation of Equation (10) then resembles the ternary relation of the Routley \& Meyer semantics. This is not surprising since the Routley \& Meyer semantics generalizes the Urquhart semantics (they are essentially the same, since as we explained it in the previous section, an operational frame is a Routley \& Meyer frame where $\mathscr{R} x y z$ holds if and only if $x \sqcup y=z$ ). Viewed from the perspective of DEL, the ternary relation then represents a particular sort of update. With this interpretation in mind, $\mathscr{R} x y z$ reads as 'the occurrence of event $y$ in world $x$ results in the world $z$ ' and the corresponding conditional $\alpha \supset \varphi$ reads as 'the occurrence in the current world of an event satisfying property $\alpha$ results in a world satisfying $\varphi$ '.

The dynamic reading of the ternary relation and its corresponding conditional is very much in line with the so-called "Ramsey Test" of conditional logic. The Ramsey test can be viewed as the very first modern contribution to the logical study of conditionals and much of the contemporary work on conditional logic can be traced back to the famous footnote of Ramsey [55]. Roughly, it consists in defining a counterfactual conditional in terms of belief revision: an agent currently believes that $\varphi$ would be true if $\psi$ were true (i.e. $\psi \supset \varphi$ ) if and only if he should believe $\varphi$ after learning $\psi$. A first attempt to provide truth conditions for conditionals, based on Ramsey's ideas, was proposed by Stalnaker. He defined his semantics by means of selection functions over possible worlds $f: W \times 2^{W} \rightarrow W$. As one can easily notice, Stalnaker's selection functions could also be considered from a formal point of view as a special kind of ternary relation, since a relation $\mathscr{R}_{f} \subseteq W \times 2^{W} \times W$ can be canonically associated to each selection function $f$. Moreover, like the ternary relation corresponding to a product update (Equation (10)), this ternary relation is 'two-sorted': the antecedent of a conditional takes value in a set of worlds (instead of a single world). ${ }^{2}$ So, the dynamic reading of the ternary semantics is consistent with the dynamic reading of conditionals proposed by Ramsey.

This dynamic reading was not really considered and investigated by substructural logicians when they connected the substructural ternary semantics with conditional logic [18]. On the other hand, the dynamic reading of inferences has been stressed to a large extent by van Benthem [27, 30] (we will come back to this point in Section 4.2), and also by Baltag and Smets who distinguished dynamic belief revision from static (standard) belief revision [12, 13, 14]. What distinguishes dynamic belief revision from static belief revision is that the latter is a revision of the agent's beliefs about the state of the world as it was before an event, and the former is a revision of the state of the world as it is after the event. Note, however, that this important distinction between static belief revision and dynamic belief revision collapses in the case of relevant logic, because in that case we only deal with propositional formulas. This shows again that a dynamic interpretation of the ternary semantics of substructural logic is consistent with the interpretations proposed by substructural logicians.

\footnotetext{
${ }^{2}$ Note that Burgess [35] already proposed a ternary semantics for conditionals, but his truth conditions and his interpretation of the ternary relation were quite different from ours.
} 
To summarize our discussion, the DEL product update provides substructural logics with an intuitive and consistent interpretation of its ternary relation. This interpretation is consistent in the sense that the intuitions underlying the definitions of the DEL framework are coherent with those underlying the ternary semantics of substructural logic, as witnessed by our quotes and citations from the substructural literature.

\section{Other interpretations of the ternary relation}

One interpretation, due to Barwise [15] and developed by Restall [56], takes worlds to be 'sites' or 'channels', a site being possibly a channel and a channel being possibly a site. If $x, y$ and $z$ are sites, $\mathscr{R} x y z$ reads as ' $x$ is a channel between $y$ and $z$ '. Hence, if $\varphi \supset \psi$ is true at channel $x$, it means that all sites $y$ and $z$ connected by channel $x$ are such that if $\varphi$ is information available in $y$, then $\psi$ is information available in $z$. Another similar interpretation due to Mares [49] adapts Israel and Perry's theory of information [54] to the relational semantics. In this interpretation, worlds are situations in the sense of Barwise and Perry's situation semantics [16] and pieces of information - called infons - can carry information about other infons: an infon might carry the information that a red light on a mobile phone carries the information that the battery of the mobile phone is low. In this interpretation, the ternary relation $\mathscr{R}$ represents the informational links in situations: if there is an informational link in situation $x$ that says that an infon $\sigma$ carries the information that the infon $\pi$ also holds, then if $\mathscr{R} x y z$ holds and $y$ contains the infon $\sigma$, then $z$ contains the infon $\pi$. Other interpretations of the ternary relation have been proposed in [18], with a particular focus on their relation to conditionality.

\section{DEL is a Substructural Logic}

In this section, we will extend the languages $\mathscr{L}$ and $\mathscr{L}_{\alpha}$ of Section 2 with the substructural operators $\circ, \supset$ and $\subset$. We will also provide a substructural semantics for this language based on the idea to view an update as a ternary relation of a substruc-

tural frame ( $\mathscr{L}_{\text {Sub }}$-model). This idea is motivated and intuitively grounded in the analysis of the previous section.

\subsection{An Extended DEL Language}

Our language extends both the language $\mathscr{L}$ and the language $\mathscr{L}_{\alpha}$ of Section 2. Like our semantics, it is two-sorted: it contains both formulas of $\mathscr{L}$ and formulas of $\mathscr{L}_{\alpha}$.

Definition 12 (Language $\mathscr{L}_{\mathscr{R}}$ ). The language $\mathscr{L}_{\mathscr{R}}$ is two-sorted and is defined by a double induction as follows: 


$$
\begin{array}{lll|l|l|l|l|l|l}
\mathscr{L}_{\mathscr{R}}^{1}: & \varphi::=p & \neg \varphi & \varphi \wedge \varphi & \varphi \vee \varphi & \square_{j} \varphi & \alpha \supset \varphi \mid \varphi \circ \alpha \\
\mathscr{L}_{\mathscr{R}}^{2}: & \alpha & :=p_{\psi} & \neg \alpha & \alpha \wedge \alpha & \alpha \vee \alpha & \square_{j} \alpha & \varphi \subset \varphi &
\end{array}
$$

where $p$ ranges over $A T M, \psi$ ranges over $\mathscr{L}_{\mathscr{R}}^{1}$ and $j$ over $A G T$. The abbreviations $\varphi \rightarrow \psi, \varphi \leftrightarrow \psi$ and $\alpha \rightarrow \beta, \alpha \leftrightarrow \beta$ are defined as in Definitions 1 and 4 .

Definition $13\left(\mathscr{L}_{\mathscr{R}}\right.$-structure and $\mathscr{L}_{\mathscr{R}}$-sequent). The $\mathscr{L}_{\mathscr{R}}$-structures are defined inductively as follows:

$$
\begin{array}{lll|l|l}
\mathscr{S}_{1}: & X & := & \varphi & (X, X) \\
\mathscr{S}_{2}: & X & := & \left(X ; X_{\alpha}\right)
\end{array}
$$

where $\varphi$ ranges over $\mathscr{L}_{\mathscr{R}}$ and $X_{\alpha}$ ranges over $\mathscr{L}_{\alpha}$-structures. A $\mathscr{L}_{\mathscr{R}}$-sequent is a $\mathscr{L}_{\alpha}$-sequent or an expression of the form $X \vdash Y$, where $X \in \mathscr{S}_{1}, Y \in \mathscr{S}_{2}$.

Definition 14 (DEL product update model). The DEL product update model is the tuple $\mathscr{M}_{\otimes}=\left(\mathscr{P}, \mathscr{R}_{1}, \ldots, \mathscr{R}_{m}, \mathscr{R}_{\otimes}, \mathscr{I}\right)$ where:

- $\mathscr{P}:=\left(\mathscr{C} \cup \mathscr{C}_{\alpha}, \leftrightarrows\right)$ where $\leftrightarrow$ is the bisimilarity relation;

- $\mathscr{R}_{j} \subseteq \mathscr{P} \times \mathscr{P}$ is a positive two-place accessibility relation on $\mathscr{P}$ for each $j \in$ $A G T$ such that for all $x, y \in \mathscr{P}$, where $x=\left(\mathscr{M}_{x}, w_{x}\right)$ and $y=\left(\mathscr{M}_{y}, w_{y}\right)$ :

$$
x \in \mathscr{R}_{j}(y) \text { iff } \mathscr{M}_{x}=\mathscr{M}_{y} \text { and } w_{x} \in R_{j}\left(w_{y}\right)
$$

- $\mathscr{R}_{\otimes}:=\left\{(x, y, z) \in \mathscr{C} \times \mathscr{C}_{\alpha} \times \mathscr{C} \mid x \otimes y=z\right\}$ is a plump ternary relation on $\mathscr{P}$;

- $\mathscr{I}(x):=I(x)$, for all $x \in \mathscr{C} \cup \mathscr{C}_{\alpha}$.

The DEL product update model is a $\mathscr{L}_{\text {Sub }}$-model where points are pointed $\mathscr{L}-$ models and pointed $\mathscr{L}_{\alpha}$-models. The ternary relation $\mathscr{R}_{\otimes}$ is defined and motivated by the explanations of the previous section. Note that the accessibility relations $R_{j}$ of $\mathscr{L}$-models and $\mathscr{L}_{\alpha}$-models are seen in this definition as positive two-place accessibility relations $\mathscr{R}_{j}$. The truth conditions are the same as the ones for $\mathscr{L}_{\mathscr{R}}$-models:

Definition 15 (Truth conditions of $\mathscr{L}_{\mathscr{R}}$ ). Let $\mathscr{M}_{\otimes}$ be the DEL product update model, $x \in \mathscr{M}_{\otimes}$ and $\varphi \in \mathscr{L}_{\mathscr{R}}$. The relation $\mathscr{M}_{\otimes}, x \| \varphi$ is defined inductively as follows:

$$
\begin{aligned}
& \mathscr{M}_{\otimes}, x \Vdash p \quad \text { iff } \quad p \in \mathscr{I}(x) \\
& \mathscr{M}_{\otimes}, x \| \neg \varphi \quad \text { iff } \quad \text { not } \mathscr{M}_{\otimes}, x \| \varphi \\
& \mathscr{M}_{\otimes}, x \| \varphi \wedge \psi \quad \text { iff } \quad \mathscr{M}_{\otimes}, x \| \varphi \text { and } \mathscr{M}_{\otimes}, x \| \psi \\
& \mathscr{M}_{\otimes}, x \| \varphi \vee \psi \quad \text { iff } \quad \mathscr{M}_{\otimes}, x \| \varphi \text { or } \mathscr{M}_{\otimes}, x \| \psi \\
& \mathscr{M}_{\otimes}, x \| \square_{j} \varphi \quad \text { iff } \quad \text { for all } y \in \mathscr{P} \text { such that } x \mathscr{R}_{j} y, \mathscr{M}_{\otimes}, y \| \varphi \\
& \mathscr{M}_{\otimes}, x \| \alpha \supset \psi \quad \text { iff } \quad \text { for all } y, z \in \mathscr{P} \text { such that } \mathscr{R}_{\otimes} x y z \text {, if } \mathscr{M}_{\otimes}, y \|-\alpha \text { then } \mathscr{M}_{\otimes}, z \| \psi \\
& \mathscr{M}_{\otimes}, x \| \psi \subset \varphi \quad \text { iff } \quad \text { for all } y, z \in \mathscr{P} \text { such that } \mathscr{R}_{\otimes} y x z \text {, if } \mathscr{M}_{\otimes}, y \| \varphi \text { then } \mathscr{M}_{\otimes}, z \| \psi \\
& \mathscr{M}_{\otimes}, x \| \varphi \circ \alpha \quad \text { iff } \quad \text { there are } y, z \in \mathscr{P} \text { such that } \mathscr{R}_{\otimes} y z x, \mathscr{M}_{\otimes}, y \| \varphi \text { and } \mathscr{M}_{\otimes}, z \| \alpha
\end{aligned}
$$

We extend the scope of the relation $\Vdash$ to also relate points to $\mathscr{L}_{\mathscr{R}}$-structures: 
$\mathscr{M}_{\otimes}, x \| X, Y \quad$ iff $\quad \mathscr{M}_{\otimes}, x \| X$ and $\mathscr{M}_{\otimes}, x \| Y$

$\mathscr{M}_{\otimes}, x \| X ; Y \quad$ iff $\quad$ there are $y, z \in \mathscr{M}_{\mathscr{R}}$ such that $\mathscr{R} y z x, \mathscr{M}_{\otimes}, y \| X$ and $\mathscr{M}_{\otimes}, z \| Y$

Let $C \subseteq \mathscr{C} \cup \mathscr{C}_{\alpha}$ be a class of pointed $\mathscr{L}$-models or $\mathscr{L}_{\alpha}$-models, and let $X \vdash \varphi$ be a $\mathscr{L}_{\mathscr{R}}$-sequent. We say that $X$ entails $\varphi$ in the class $C$, written $X \|_{C} \varphi$, when the following holds:

$$
X \|_{C} \varphi \quad \text { iff } \quad \text { for all } x \in C \text {, if } \mathscr{M}_{\otimes}, x \Vdash X \text { then } \mathscr{M}_{\otimes}, x \Vdash \varphi .
$$

We also write $X \| \varphi$ for $X \|_{\mathscr{\mathscr { C }} \cup \mathscr{C}_{\alpha}} \varphi$.

Naturally, the truth conditions for $\Vdash$ coincide with the truth conditions for $\models$ if we only consider epistemic or event formulas:

Proposition 1. Let $\mathscr{M}_{\otimes}$ be the DEL product update model, $\varphi \in \mathscr{L}$ and $x \in \mathscr{M}_{\otimes}$ such that $x \in \mathscr{C}$. Then, $\mathscr{M}_{\otimes}, x \Vdash \varphi$ iff $x \models \varphi$. Let $\alpha \in \mathscr{L}_{\alpha}$ and let $y \in \mathscr{M}_{\otimes}$ such that $y \in \mathscr{C}_{\alpha}$. Then, $\mathscr{M}_{\otimes}, y \| \alpha$ iff $y \models \alpha$.

Remark 1 . The frame semantics of substructural logic is very abstract and general and it provides a rich framework which captures a wide range of logics, such as arrow logic [23, Chap. 8], action frames and domain space (see [57, Example 11.1211.15] for more details). But the epistemic temporal models of ETL [53] (which have been related to DEL in $[33,32]$ ) can also be viewed as models of the ternary semantics of substructural logic [5].

\subsection{DEL Operators are Substructural Operators}

In this section, we will show that the DEL operators introduced in $[3,4]$ correspond to the substructural operators $\circ, \supset$ and $\subset$. We will also relate the work of van Benthem on dynamic inference with the DEL-sequents of $[3,4,7]$.

\subsubsection{Dynamic Inferences and DEL-sequents}

\section{Dynamic Inferences}

In the so-called 'dynamic turn', van Benthem was interested in various dynamic styles of inference where propositions are procedures changing information states. These dynamic styles of inference differ greatly from the classical Tarskian's valid inferences because the latter are supposed to transmit and preserve truth. Among various dynamic styles of inference (such as the so-called test-test, update-update or update-test consequence $[21,23,51]$ ), he studied the concrete following one, which can be defined within the DEL framework:

Definition 16 (Dynamic inference [25]). Let $\varphi_{0}, \varphi_{1}, \ldots, \varphi_{n}, \psi \in \mathscr{L}$. We define the dynamic inference $\varphi_{0}, \varphi_{1}, \ldots, \varphi_{n} \models \psi$ as follows: 


$$
\begin{aligned}
\varphi_{1}, \ldots, \varphi_{n} \models \varphi \quad \text { iff } & \text { for all pointed } \mathscr{L} \text {-model }(\mathscr{M}, w), \text { and public announce- } \\
& \text { ment } \mathscr{L}_{\alpha} \text {-models }\left(\mathscr{E}_{1}, e_{1}\right), \ldots,\left(\mathscr{E}_{n}, e_{n}\right) \text { of } \varphi_{1}, \ldots, \varphi_{n} \text { re- } \\
& \text { spectively, }(\mathscr{M}, w) \otimes\left(\mathscr{E}_{1}, e_{1}\right) \otimes \ldots \otimes\left(\mathscr{E}_{n}, e_{n}\right) \models \varphi .
\end{aligned}
$$

van Benthem noticed that various dynamic styles of inference obey structural rules of inference which are non-classical. For example, all the structural rules of classical logic of Figure 5 fail for dynamic inference, but the structural rules below characterize completely the dynamic inference [25] (below, $\vec{\varphi}$ stands for $\varphi_{1}, \ldots, \varphi_{n}$ and $\vec{\psi}$ stands for $\psi_{1}, \ldots, \psi_{n}$, where $\left.\varphi_{1}, \ldots, \varphi_{n}, \psi_{1}, \ldots, \psi_{n} \in \mathscr{L}\right)$ :

$$
\begin{array}{lr}
\text { if } \vec{\varphi} \models \varphi \text { then } \psi, \vec{\varphi} \models \varphi & \text { (Left-Monotonicity) } \\
\text { if } \vec{\varphi} \models \varphi \text { and } \vec{\varphi}, \varphi, \vec{\psi}=\psi \text { then } \vec{\varphi}, \vec{\psi} \models \psi & \text { (Left-Cut) } \\
\text { if } \vec{\varphi} \models \varphi \text { and } \vec{\varphi}, \vec{\psi} \models \psi \text { then } \vec{\varphi}, \varphi, \vec{\psi} \models \psi & \text { (Cautious Monotonicity) }
\end{array}
$$

\section{DEL-sequents}

In [3], I introduced what I called DEL-sequents. They are a particular sort of dynamic inference and are defined as follows:

Definition 17 (DEL-sequent [3]). Let $\varphi, \varphi_{f} \in \mathscr{L}$ and $\alpha \in \mathscr{L}_{\alpha}$. We define the logical consequence relation $\varphi, \alpha=\varphi_{f}$ as follows:

$\varphi, \alpha=\varphi_{f} \quad$ iff $\quad$ for all pointed $\mathscr{L}$-model $(\mathscr{M}, w)$, all $\mathscr{L}_{\alpha}-\operatorname{model}(\mathscr{E}, e)$ such that $\mathscr{M}, w \models I(e), \mathscr{M}, w \models \varphi$ and $\mathscr{E}, e \models \alpha$, it holds that $(\mathscr{M}, w) \otimes$ $(\mathscr{E}, e) \models \varphi_{f}$.

In [7], DEL-sequents are generalized to take into account sequences of events and not only 'one-shot' occurrence of events. Several generalized DEL-sequents are introduced in [7] but they are all reducible to the following one:

Definition 18 (Generalized DEL-sequent [7]). Let $\varphi_{0}, \ldots, \varphi_{n} \in \mathscr{L}$, let $\alpha_{1}, \ldots, \alpha_{n} \in$ $\mathscr{L}_{\alpha}$ and let $\psi \in \mathscr{L}$. Then,

$$
\varphi_{0}, \alpha_{1}, \varphi_{1}, \ldots, \alpha_{n}, \varphi_{n} \models \psi
$$

iff

if for all pointed $\mathscr{L}$-model $(\mathscr{M}, w)$, and $\mathscr{L}_{\alpha}$-models $\left(\mathscr{E}_{1}, e_{1}\right), \ldots,\left(\mathscr{E}_{n}, e_{n}\right)$ such that for all $i \in\{1, \ldots, n\}, \mathscr{E}_{i}, e_{i}=\alpha_{i},(\mathscr{M}, w) \otimes\left(\mathscr{E}_{1}, e_{1}\right) \otimes \ldots \otimes\left(\mathscr{E}_{i}, e_{i}\right)$ is defined and makes $\varphi_{i}$ true, then it holds that $(\mathscr{M}, w) \otimes\left(\mathscr{E}_{1}, e_{1}\right) \otimes \ldots \otimes$ $\left(\mathscr{E}_{n}, e_{n}\right) \models \psi$.

As one can easily notice, dynamic inferences can be translated into DELsequents if we resort to the common knowledge/belief operator $\square_{A G T}^{*} \varphi$ (see for example [41] for a definition and a detailed study of this operator):

Proposition 2. Let $\varphi_{0}, \varphi_{1}, \ldots, \varphi_{n}, \varphi \in \mathscr{L}$. Then, the following holds:

$$
\varphi_{1}, \ldots, \varphi_{n} \models \varphi \quad \text { iff } \quad \top, p_{\varphi_{1}} \wedge \square_{A G T}^{*} p_{\varphi_{1}}, \ldots, \top, p_{\varphi_{n}} \wedge \square_{A G T}^{*} p_{\varphi_{n}}, \top \models \varphi \wedge \square_{A G T}^{*} \varphi
$$


Thus, DEL-sequents are more expressive than dynamic inferences, and also more abstract because they 'operate' at a deeper level, a semantical one. It is this more general and abstract approach towards dynamic styles of inference that will allow us to relate more precisely and closely DEL with substructural logics, and explain to a certain extent why the substructural phenomena occurring in dynamic inferences and observed by van Benthem arise.

\subsubsection{DEL-sequents for Progression, Regression and Epistemic Planning}

Substructural logics and dynamic logics of information flow are long-standing interests of van Benthem [22, 23, 25, 27, 29, 30]. Recently again [29], he expressed some worries about interpreting the Lambek Calculus (the paradigmatic substructural logic) as a base logic of information flow while trying to connect the operators $\circ, \supset$ and $\subset$ of substructural logic to some sort of DEL operators. Indeed, the DEL operators usually rely on the regular algebra of sequential composition, choice and iteration which are of a quite different nature. Recently, I introduced some DEL operators called progression, regression and epistemic planning [3, 4], the operator of regression being a natural generalization of the standard and original action modality $[\mathscr{E}, e] \varphi$ of DEL [17]. It turns out that these operators can all be identified with connectives of the substructural language $\mathscr{L}_{\mathscr{R}}$. We first briefly recall their definitions below and then we give our correspondence results between the two kinds of operators.

\section{Progression}

The operator of progression is denoted $\otimes$ in [3]. In [4, Def. 41], a constructive definition of this operator is provided using characteristic formulas (called "Kit Fine" formulas). Here, we provide an alternative and non-constructive definition of the progression of $\varphi$ by $\alpha$, denoted $\varphi \otimes \alpha$ :

Theorem 1. Let $\left(\mathscr{M}_{f}, w_{f}\right)$ be a pointed $\mathscr{L}$-model and let $\varphi \in \mathscr{L}$ and $\alpha \in \mathscr{L}_{\alpha}$. Then,

$$
\begin{aligned}
\mathscr{M}_{f}, w_{f}=\varphi \otimes \alpha \quad \text { iff } \quad & \text { there is a pointed } \mathscr{L} \text {-model }(\mathscr{M}, w) \text { and a pointed } \\
& \mathscr{L}_{\alpha} \text {-model }(\mathscr{E}, e) \operatorname{such} \text { that }(\mathscr{M}, w) \otimes(\mathscr{E}, e) \leftrightarrows\left(\mathscr{M}_{f}, w_{f}\right), \\
& \mathscr{M}, w \models \varphi \text { and } \mathscr{E}, e \models \alpha
\end{aligned}
$$

Proof. It follows from Lemmata 43 and 44 of [3].

\section{Epistemic Planning}

The operator of epistemic planning is denoted $Q_{P}$ in [4]. It is defined relatively to a finite set $P$ of formulas/preconditions/atomic events. In [4, Def. 14-15], a construc- 
tive definition of this operator is provided using characteristic formulas (called "Kit Fine" formulas). As it turns out, an alternative and non-constructive definition of the epistemic planning from $\varphi$ to $\varphi_{f}$, denoted $\varphi \otimes_{P} \varphi_{f}$, exists as well:

Theorem 2 ([4]). Let $\varphi, \varphi_{f} \in \mathscr{L}$ and let $P$ be a finite subset of $\mathscr{L}$. Then, for all $P$-complete $\mathscr{L}_{\alpha}-$ model $(\mathscr{E}, e)$, it holds that

$$
\mathscr{E}, e \models \varphi \otimes_{P} \varphi_{f} \quad \text { iff } \quad \begin{aligned}
& \text { there is }(\mathscr{M}, w) \text { such that } \mathscr{M}, w \models \varphi, \\
& \mathscr{M}, w \models I(e) \text { and }(\mathscr{M}, w) \otimes(\mathscr{E}, e) \models \varphi_{f}
\end{aligned}
$$

The dual of the operator $\varphi \otimes_{P} \varphi_{f}$ is defined by:

$$
\varphi[\otimes]_{P} \varphi_{f}:=\neg\left(\varphi \otimes_{P} \neg \varphi_{f}\right)
$$

Theorem 2 entails that $\varphi[\Theta]_{P} \varphi_{f}$ can be alternatively defined as follows: for all $P$-complete $\mathscr{L}_{\alpha}$-model $(\mathscr{E}, e)$, it holds that

$$
\begin{aligned}
\mathscr{E}, e \models \varphi[\theta]_{P} \varphi_{f} \quad \text { iff for all }(\mathscr{M}, w) \text { such that } \mathscr{M}, w \models \varphi \text {, if } \\
\qquad \mathscr{M}, w \models I(e) \text { then }(\mathscr{M}, w) \otimes(\mathscr{E}, e) \models \varphi_{f}
\end{aligned}
$$

Example 4. In the situation depicted in the $\mathscr{L}$-model of Figure 1, agent B does not know that agent $\mathrm{A}$ has the red card and does not know that agent $\mathrm{C}$ has the blue card: $\mathscr{M}, w \models\left(\diamond_{B} r_{A} \wedge \diamond_{B} \neg r_{A}\right) \wedge\left(\diamond_{B} b_{C} \wedge \diamond_{B} \neg b_{C}\right)$. Our problem is therefore the following:

What sufficient and necessary property (i.e. 'minimal' property) an event should fulfill so that its occurence in the initial situation $(\mathscr{M}, w)$ results in a situation where agent $\mathrm{B}$ knows the true state of the world, i.e. agent B knows that agent A has the red card and that agent $C$ has the blue card?

The answer to this question obviously depends on the kind of atomic events we consider. In this example, the events $P=\left\{p_{b_{C}}, p_{r_{A}}, p_{w_{B}}\right\}$ under consideration are the following. First, agent $\mathrm{C}$ shows her blue card $\left(p_{b_{C}}\right)$, second, agent A shows her red card $\left(p_{r_{A}}\right)$, and third, agent $\mathrm{B}$ herself shows her white card $\left(p_{w_{B}}\right)$. Answering this question amounts to compute the formula $(M, w) \Theta_{P} \square_{B}\left(r_{A} \wedge b_{C} \wedge w_{B}\right)$. Applying the algorithm of [4, Definition 15], we obtain that

$$
(\mathscr{M}, w) \otimes_{P} \square_{B}\left(r_{A} \wedge b_{C} \wedge w_{B}\right) \leftrightarrow \square_{B}\left(p_{b_{C}} \vee p_{r_{A}}\right) \text { is valid. }
$$

In other words, this result states that agent $\mathrm{B}$ should believe either that agent $\mathrm{A}$ shows her red card or that agent $C$ shows her blue card in order to know the true state of the world. Indeed, since there are only three different cards which are known by the agents and agent B already knows her card, if she learns the card of (at least) one of the other agents, she will also be able to infer the card of the third agent.

\section{Regression}

The operator of regression is denoted $\oslash$ in [3]. In [4, Def. 41], a constructive definition of this operator is provided using characteristic formulas (called "Kit Fine" 
formulas) by adapting and translating the reduction axioms of [17]. As it turns out, an alternative and non-constructive definition of the regression of $\varphi_{f}$ by $\alpha$, denoted $\alpha \oslash \varphi_{f}$, exists as well:

Theorem 3. Let $\alpha \in \mathscr{L}_{\alpha}$ and $\varphi_{f} \in \mathscr{L}$. Then, for all $\mathscr{L}$-model $(\mathscr{M}, w)$, it holds that

$$
\mathscr{M}, w \models \alpha \oslash \varphi_{f} \quad \text { iff } \quad \begin{aligned}
& \text { there is }(\mathscr{E}, e) \text { such that } \mathscr{E}, e \models \alpha, \\
& \mathscr{M}, w \models I(e) \text { and }(\mathscr{M}, w) \otimes(\mathscr{E}, e) \models \varphi_{f}
\end{aligned}
$$

Note that we could define a dual operator of $\alpha \oslash \varphi_{f}$ as follows:

$$
\alpha[\oslash] \varphi_{f}=\neg\left(\alpha \oslash \neg \varphi_{f}\right)
$$

Then, the counterpart of Theorem 3 for this dual operator is as follows:

$$
\mathscr{M}, w=\alpha[\oslash] \varphi_{f} \quad \text { iff } \quad \begin{aligned}
& \text { for all }(\mathscr{E}, e) \text { such that } \mathscr{E}, e \models \alpha, \\
& \quad \text { if } \mathscr{M}, w \models I(e) \text { then }(\mathscr{M}, w) \otimes(\mathscr{E}, e) \models \varphi_{f}
\end{aligned}
$$

As shown in [4, Sec. 6], the operator $\alpha[\oslash] \varphi_{f}$ is a generalization of the original and more standard DEL operator $[\mathscr{E}, e] \varphi$ almost exclusively used in the DEL literature [17].

\section{Correspondence between DEL and Substructural Operators}

As one can easily notice, there is a strong similarity between the operations of progression, epistemic planning and regression and the operations of substructural logic, more precisely of the Lambek Calculus. In fact, there exists a rigorous mapping between them, as the following theorem shows:

Theorem 4. Let $P$ be a finite subset of $\mathscr{L}$, let $x=(\mathscr{M}, w) \in \mathscr{C}$ and let $y=(\mathscr{E}, e) \in$ $\mathscr{C}_{\alpha}^{P}$ be a P-complete pointed event model. Let $\varphi, \psi \in \mathscr{L}$ and let $\alpha \in \mathscr{L}_{\alpha}$. Then,

$$
\begin{array}{lll}
\mathscr{M}_{\otimes}, x \Vdash \varphi \circ \alpha & \text { iff } & x=\varphi \otimes \alpha \\
\mathscr{M}_{\otimes}, x \| \alpha \supset \varphi & \text { iff } & x=\alpha[\oslash] \varphi \\
\mathscr{M}_{\otimes}, y \Vdash \psi \subset \varphi & \text { iff } & y=\varphi[\theta]_{P} \psi
\end{array}
$$

Moreover, for all $\alpha, \alpha_{1}, \ldots, \alpha_{n} \in \mathscr{L}_{\alpha}$, for all $\varphi, \psi, \varphi_{0}, \varphi_{1}, \ldots, \varphi_{n} \in \mathscr{L}$, we have:

$$
\begin{array}{ll}
\varphi ; \alpha \| \psi & \text { iff } \quad \varphi, \alpha \models \psi \\
\left(\left(\left(\varphi_{0} ; \alpha_{1}\right), \varphi_{1}\right) ; \ldots ; \alpha_{n}\right), \varphi_{n} \Vdash \psi & \text { iff } \quad \varphi_{0}, \alpha_{1}, \varphi_{1}, \ldots, \alpha_{n}, \varphi_{n} \models \psi
\end{array}
$$

The key Theorem 42 of [3] relates DEL-sequents and the operator of progression: for all $\varphi, \varphi_{f} \in \mathscr{L}$ and $\alpha \in \mathscr{L}_{\alpha}$, it holds that

$$
\varphi, \alpha=\varphi_{f} \text { iff } \varphi \otimes \alpha \models \varphi_{f} .
$$




\begin{tabular}{|c|c|}
\hline Substructural operators & DEL operators \\
\hline$\circ$ & $\otimes$ \\
$\supset$ & {$[\oslash]$} \\
$\subset$ & {$[\odot]$} \\
\hline
\end{tabular}

Fig. 6 Correspondence between DEL and substructural operators

As it turns out, this theorem is also valid in any substructural logic: it corresponds to the theorem of Equation (2). More generally, all the theorems of the nonassociative Lambek calculus hold in our DEL setting if we use the translation given in Figure 6. In particular, we have the following results which are the counterparts of Equations (3), (4) and (5) in our setting:

Corollary 1. Let $P$ be a finite subset of $\mathscr{L}$. For all $\varphi, \varphi_{f} \in \mathscr{L}$ and $\alpha \in \mathscr{L}_{\alpha}$, it holds that

$$
\begin{aligned}
& \varphi ; \alpha \| \varphi_{f} \quad \text { iff } \varphi \| \alpha[\oslash] \varphi_{f} \\
& \varphi \Vdash \alpha[\oslash] \varphi_{f} \quad \text { iff } \varphi \otimes \alpha \| \varphi_{f} \\
& \varphi \otimes \alpha \| \varphi_{f} \quad \text { iff } \quad \alpha \|_{\mathscr{C}_{\alpha}^{P}} \varphi[\theta]_{P} \varphi_{f} \\
& \varphi \Vdash \alpha[\oslash] \varphi_{f} \quad \text { iff } \quad \alpha \|_{\mathscr{C}_{\alpha}^{P}} \varphi[\theta]_{P} \varphi_{f}
\end{aligned}
$$

\section{Conclusion}

We proved in this article that DEL is a two-sorted substructural logic. Also, we argued in Section 3.2 that our embedding of DEL within the framework of substructural logic is intuitively consistent, in the sense that in this embedding the intuitions underlying the DEL framework are coherent with the intuitive interpretations proposed for the ternary semantics of substructural logics. This may explain to a certain extent why some substructural phenomena arise in the dynamic inferences of Section 4.2.1. As observed by van Benthem, "it seemed that structural rules address mere symptoms of some underlying phenomenon" [30, p. 297]. I claim that these "symptoms" are caused at a deeper semantic level by the fact that an update, and in that case the DEL product update, can be represented by the ternary relation of substructural logics.

In a certain sense, this article is in line with the approach of van Benthem [28, 30] and contributes to relate even more closely the research programs of Logical Pluralism [19] and Logical Dynamics [30]. Roughly, the informal idea underpinning the connection between these two logical paradigms is to consider different reasoning styles and their corresponding consequence relations as the result of different sorts of updates induced by various informational tasks (such as observation, memory, questions and answers, dialogue, or general communication). We showed in this article that this approach is not only meaningful from an intuitive point of view, but 
it can also be realized at a formal level if the ternary relation of substructural logic is interpreted intuitively as a sort of update. So, we hope that our embedding will strengthen the connections between the two areas of research represented by Logical Pluralism (and substructural logics) on the one hand and Logical Dynamics on the other hand. In fact, our point of view is also very much in line with the claim of Gärdenfors and Makinson [44, 48] that non-monotonic reasoning and belief revision are "two sides of the same coin": as a matter of fact, non-monotonic reasoning is a reasoning style and belief revision is a sort of update. Likewise, the formal connection in this case also relies on a similar idea based on the Ramsey test.

In this article, we focused our attention on the DEL product update. It is, however, a particular kind of update operator and the ternary relation of substructural logics could actually be a representation of any sort of update, including the various revision and update operators which have been studied in the logics of "common sense reasoning" of artificial intelligence and philosophical logic, such as conditional logic [52], default and non-monotonic logics [47, 42], belief revision theory [43], etc... Different kinds of updates, induced by different informational tasks, define different kinds of reasoning styles. If one adheres to our interpretation of the ternary relation, the dynamic notion of update then becomes the foundational concept of substructural logics.

This observation gives rise, in turn, to a research thread where updates are the central objects of study and where we can (re-)analyze various updates within the generic and abstract logical framework of substructural logics. This research thread is of course very much in line with van Benthem's long standing interest in information and logical dynamics, but also with his interest in modal correspondence theory, the area of logic where he first contributed [20,24]. For example, we could elicit a number of axioms and inference rules that define specific properties of updates, some being possibly satisfied by the DEL product update. In other words, we could develop a correspondence theory for analyzing and studying the notion of update similar to the correspondence theory developed by van Benthem $[20,24]$ for modal logic. A basic correspondence theory with a complete characterization of the DEL product update in terms of axioms and inference rules is given in [5].

Acknowledgements I thank Olivier Roy and Ole Hjortland for organizing and inviting me to an inspiring workshop on substructural epistemic logic in Munich in February 2013. Also, I thank Johan van Benthem and Igor Sedlar for comments on an earlier version of this article. Finally, I thank Sean Sedwards for checking the English of this article.

\section{References}

1. Aucher, G.: A combined system for update logic and belief revision. In: M. Barley, N.K. Kasabov (eds.) PRIMA, Lecture Notes in Computer Science, vol. 3371, pp. 1-17. Springer (2004)

2. Aucher, G.: Perspectives on belief and change. Ph.D. thesis, University of Otago - University of Toulouse (2008) 
3. Aucher, G.: DEL-sequents for progression. Journal of Applied Non-Classical Logics 21(3-4), 289-321 (2011)

4. Aucher, G.: DEL-sequents for regression and epistemic planning. Journal of Applied NonClassical Logics 22(4), 337-367 (2012)

5. Aucher, G.: Update Logic. Research Report RR-8341, INRIA (2013). URL http://hal.inria.fr/hal-00849856

6. Aucher, G., Herzig, A.: Exploring the power of converse events. Dynamic Formal Epistemology pp. 51-74 (2011)

7. Aucher, G., Maubert, B., Schwarzentruber, F.: Generalized DEL-sequents. In: L.F. del Cerro, A. Herzig, J. Mengin (eds.) JELIA, Lecture Notes in Computer Science, vol. 7519, pp. 54-66. Springer (2012)

8. Baltag, A., Coecke, B., Sadrzadeh, M.: Algebra and sequent calculus for epistemic actions. Electronic Notes in Theoretical Computer Science 126, 27-52 (2005)

9. Baltag, A., Coecke, B., Sadrzadeh, M.: Epistemic actions as resources. Journal of Logic and Computation 17(3), 555-585 (2007)

10. Baltag, A., Moss, L.: Logic for epistemic programs. Synthese 139(2), 165-224 (2004)

11. Baltag, A., Moss, L., Solecki, S.: The logic of public announcements, common knowledge and private suspicions. Tech. rep., Indiana University (1999)

12. Baltag, A., Smets, S.: Conditional doxastic models: A qualitative approach to dynamic belief revision. Electronic Notes in Theoretical Computer Science 165, 5-21 (2006)

13. Baltag, A., Smets, S.: Texts in Logic and Games, vol. 4, chap. The Logic of Conditional Doxastic Actions, pp. 9-31. Amsterdam University Press (2008)

14. Baltag, A., Smets, S.: Texts in Logic and Games, vol. 3, chap. A Qualitative Theory of Dynamic Interactive Belief Revision, pp. 9-58. Amsterdam University Press (2008)

15. Barwise, J.: Constraints, channels, and the flow of information. Situation theory and its applications 3, 3-27 (1993)

16. Barwise, J., Perry, J.: Situations and Attitudes. Cambridge, Massachusetts. MIT Press (1983)

17. Batlag, A., Moss, L.S., Solecki, S.: The logic of public announcements and common knowledge and private suspicions. In: I. Gilboa (ed.) TARK, pp. 43-56. Morgan Kaufmann (1998)

18. Beall, J., Brady, R., Dunn, J.M., Hazen, A., Mares, E., Meyer, R.K., Priest, G., Restall, G., Ripley, D., Slaney, J., et al.: On the ternary relation and conditionality. Journal of philosophical logic 41(3), 595-612 (2012)

19. Beall, J.C., Restall, G.: Logical pluralism. Oxford University Press (2006)

20. van Benthem, J.: Modal correspondence theory. Ph.D. thesis, University of Amsterdam (1977)

21. van Benthem, J.: General dynamics. Theoretical Linguistics 17(1-3), 159-202 (1991)

22. van Benthem, J.: Language in Action: Categories, Lambdas and Dynamic Logic, vol. 130. North Holland (1991)

23. van Benthem, J.: Exploring logical dynamics. CSLI publications Stanford (1996)

24. van Benthem, J.: Correspondence theory. Handbook of philosophical logic 3, 325-408 (2001)

25. van Benthem, J.: Meaning: the Dynamic Turn, chap. Structural Properties of Dynamic Reasoning, pp. 15-31. Elsevier, Amsterdam (2003)

26. van Benthem, J.: Dynamic logic for belief revision. Journal of Applied Non-Classical Logics 17(2), 129-155 (2007)

27. van Benthem, J.: Inference in action. Publications de l'Institut Mathématique-Nouvelle Série 82(96), 3-16 (2007)

28. van Benthem, J.: Logical dynamics meets logical pluralism? The Australasian Journal of Logic 6, 182-209 (2008)

29. van Benthem, J.: Modal logic for open minds. CSLI publications (2010)

30. van Benthem, J.: Logical Dynamics of Information and Interaction. Cambridge University Press (2011)

31. van Benthem, J.: McCarthy variations in a modal key. Artificial intelligence 175(1), 428-439 (2011)

32. van Benthem, J., Gerbrandy, J., Hoshi, T., Pacuit, E.: Merging frameworks for interaction. Journal of Philosophical Logic 38(5), 491-526 (2009). DOI 10.1007/s10992-008-9099-x. URL http://dx.doi.org/10.1007/s10992-008-9099-x 
33. van Benthem, J., Gerbrandy, J., Pacuit, E.: Merging frameworks for interaction: DEL and ETL. In: D. Samet (ed.) Theoretical Aspect of Rationality and Knowledge (TARK XI), pp. 72-82. Brussels (2007)

34. van Benthem, J., Kooi, B.: Reduction axioms for epistemic actions. In: R. Schmidt, I. PrattHartmann, M. Reynolds, H. Wansing (eds.) AiML-2004: Advances in Modal Logic, number UMCS-04-9-1 in Technical Report Series, pp. 197-211. University of Manchester (2004)

35. Burgess, J.P.: Quick completeness proofs for some logics of conditionals. Notre Dame Journal of Formal Logic 22(1), 76-84 (1981)

36. van Ditmarsch, H.: Prolegomena to dynamic logic for belief revision. Synthese 147, 229-275 (2005)

37. van Ditmarsch, H., van der Hoek, W., Kooi, B.: Dynamic Epistemic Logic, Synthese library, vol. 337. Springer (2007)

38. van Ditmarsch, H.P., Herzig, A., Lima, T.D.: From situation calculus to dynamic epistemic logic. Journal of Logic and Computation 21(2), 179-204 (2009)

39. Dunn, J.M., Restall, G.: Relevance logic. Handbook of philosophical logic 6, 1-128 (2002)

40. van Eijck, J.: Reducing dynamic epistemic logic to PDL by program transformation. Tech. Rep. SEN-E0423, CWI (2004)

41. Fagin, R., Halpern, J., Moses, Y., Vardi, M.: Reasoning about knowledge. MIT Press (1995)

42. Gabbay, D.M., Hogger, C.J., Robinson, J.A., Siekmann, J., Nute, D. (eds.): Handbook of logic in artificial intelligence and logic programming, vol. Nonmonotonic reasoning and uncertain reasoning (Volume 3). Clarendon Press (1998)

43. Gärdenfors, P.: Knowledge in Flux (Modeling the Dynamics of Epistemic States). Bradford/MIT Press, Cambridge, Massachusetts (1988)

44. Gärdenfors, P.: Belief revision and nonmonotonic logic: Two sides of the same coin? In: Logics in AI, pp. 52-54. Springer (1991)

45. Hintikka, J.: Knowledge and Belief, An Introduction to the Logic of the Two Notions. Cornell University Press, Ithaca and London (1962)

46. Liu, F.: Changing for the better: Preference dynamics and agent diversity. Ph.D. thesis, ILLC, University of Amsterdam (2008)

47. Makinson, D.: Bridges from classical to nonmonotonic logic. King's College (2005)

48. Makinson, D., Gärdenfors, P.: Relations between the logic of theory change and nonmonotonic logic. In: A. Fuhrmann, M. Morreau (eds.) The Logic of Theory Change, Lecture Notes in Computer Science, vol. 465, pp. 185-205. Springer (1989)

49. Mares, E.D.: Relevant logic and the theory of information. Synthese 109(3), 345-360 (1996)

50. Mares, E.D., Meyer, R.K.: The Blackwell guide to philosophical logic, chap. Relevant Logics. Wiley-Blackwell (2001)

51. Muskens, R., van Benthem, J., Visser, A.: Handbook of logic and language, chap. Dynamics, pp. 607-670. Elsevier (2011)

52. Nute, D., Cross, C.B.: Handbook of philosophical logic, vol. 4, chap. Conditional logic, pp. 1-98. Kluwer Academic Pub (2001)

53. Parikh, R., Ramanujam, R.: A knowledge based semantics of messages. Journal of Logic, Language and Information 12(4), 453-467 (2003)

54. Perry, J., Israel, D.: What is information? Information, Language, and Cognition 1 (1990)

55. Ramsey, F.: Philosophical Papers, chap. General Propositions and Causality. Cambridge University Press, Cambridge (1929)

56. Restall, G.: Information flow and relevant logics. In: Logic, Language and Computation: The 1994 Moraga Proceedings. CSLI, pp. 463-477. csli Publications (1996)

57. Restall, G.: An Introduction to Substructural Logics. Routledge (2000)

58. Restall, G.: Relevant and substructural logics. Handbook of the History of Logic 7, 289-398 (2006)

59. Routley, R., Meyer, R.: The semantics of entailment. Studies in Logic and the Foundations of Mathematics 68, 199-243 (1973)

60. Routley, R., Meyer, R.K.: The semantics of entailment-ii. Journal of Philosophical Logic 1(1), 53-73 (1972) 
61. Routley, R., Meyer, R.K.: The semantics of entailment-iii. Journal of philosophical logic 1(2), 192-208 (1972)

62. Routley, R., Plumwood, V., Meyer, R.K.: Relevant logics and their rivals. Ridgeview Publishing Company (1982)

63. Urquhart, A.: A general theory of implication. Journal of Symbolic Logic 37(443), 270 (1972)

64. Urquhart, A.: Semantics for relevant logics. Journal of Symbolic Logic pp. 159-169 (1972)

65. Urquhart, A.I.: Completeness of weak implication. Theoria 37(3), 274-282 (1971) 